\title{
CORRESPONDENCE
}

\section{Cutaneous Crohn's disease presenting as genital warts: successful treatment with adalimumab}

A 21-year-old female patient presented with a 1-year history of multiple polypoid skin tags in the perianal area, previously diagnosed as genital warts. She had been treated with cryotherapy and imiquimod for 6 months without any clinical improvement. Her medical history included the diagnosis of ileocecal Crohn's disease (CD), made 2 years earlier. CD activity index (CDAI) was 231 with an inadequate response to systemic therapy (corticosteroids, sulfasalazine, azathioprine and metronidazole). Risk factors for sexually transmitted infections (STIs) were not identified. Clinical examination revealed multiple polypoid and edematous skin tags in the perianal region and an erythematous and tender plaque in the left perianal area, extending to the homolateral buttock (figures $1 A, B$ ). Proctologic examination and imaging studies showed an anal tumefaction below the dentate line and excluded the presence of perianal ulceration, abscesses and fistulas. Laboratory evaluation revealed ferropenic anemia (haemoglobin $8.8 \mathrm{~g} / \mathrm{dL}$, iron $15 \mu \mathrm{g} / \mathrm{dL}$, ferritin $1.6 \mathrm{ng} / \mathrm{mL}$ ), and elevation of inflammatory parameters (C-reactive protein $4.1 \mathrm{~g} / \mathrm{dL}$; sedimentation rate $62 \mathrm{~mm} / \mathrm{h}$ ). STIs were also excluded. An excisional biopsy of a perianal polypoid structure and a punch biopsy from the plaque of left buttock were performed. Both specimens revealed non-caseating epithelioid granulomas and multinucleated giant cells through the dermis, supporting the diagnosis of perianal cutaneous CD (figure 1D). Infectious granulomatous skin disorders were excluded. Treatment with adalimumab was started with $80 \mathrm{mg}$ loading dose at week 0 and then $40 \mathrm{mg}$ every other week. Six weeks later, intestinal clinical remission was achieved (CDAI 72). A decrease in the erythema and induration of the cutaneous perianal lesions was seen after the second week of adalimumab treatment, followed by a progressive decrease in the size of the perianal skin tags. A significant improvement was achieved, although not complete (figure 1C). Currently, after 60 weeks of adalimumab monotherapy, she remains clinically stable.

$\mathrm{CD}$ is associated with a great variety of cutaneous lesions, which can be classified as contiguous granulomatous (oral and perianal) lesions, non-contiguous CD lesions, reactive skin eruptions and lesions secondary to nutritional deficiencies [1]. Perianal CD refers to involvement of the anal region, most commonly presenting with abscesses and fistulas, although fissures, strictures, moist plaques and edematous skin tags may be also present [2]. Among mucocutaneous manifestations of $\mathrm{CD}$, contiguous perianal skin lesions are the most frequent findings, exhibiting an extremely variable macroscopic appearance [3]. Pseudocondylomatous morphologies with granulomatous inflammation are an uncommon perianal manifestation of $\mathrm{CD}$ and may lead to misdiagnosis with genital warts $[4,5]$. Therefore, a delay in diagnosis is frequent and was also described in two previous published cases ( 3 and 4 months). However, in contrast to the case described by Asma et al. [4] and the paediatric case described by Gard et al. [5], in which the diagnosis of luminal $\mathrm{CD}$ was made after the onset of perianal polypoid lesions, in our patient the diagnosis of ileocecal CD was already known. A variable response to therapy was also seen, with an excellent response to medical treatment in the former case and resistance to antibiotics, immunosuppressive agents and infliximab in the later.

Cutaneous CD is frequently refractory to conventional therapies and treatment of the gastrointestinal disease does not necessarily result in improvement of the skin lesions [3]. Recently, a clinical response to anti-TNF antagonists, particulary to infliximab, has been reported $[1,3]$; however, the efficacy of adalimumab in cutaneous $\mathrm{CD}$ is seldom described in the literature [6].

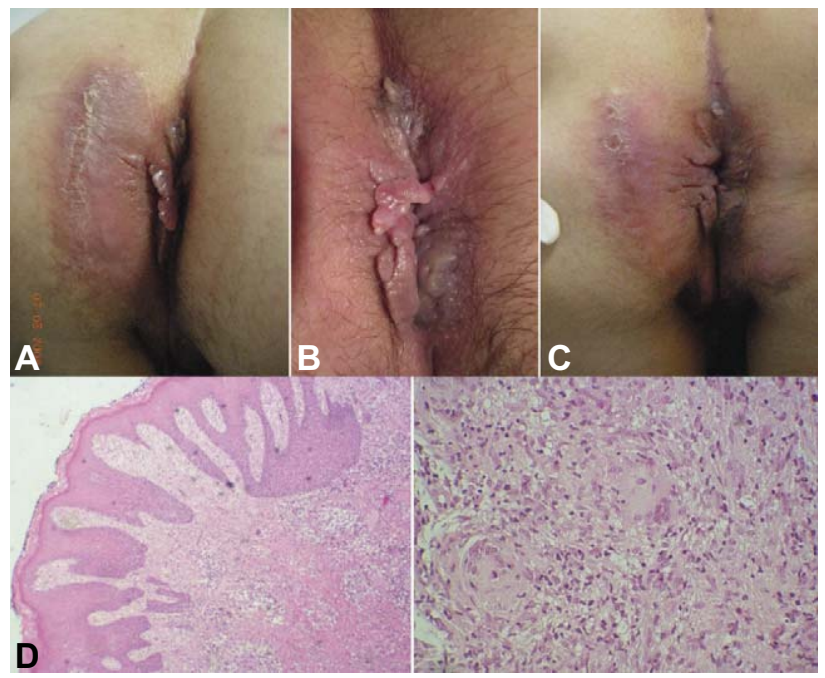

Figure 1. A) Perianal skin tags and erythematous plaque located on left buttock. B) Detail view of edematous and polypoid skin tags. C) Significant clinical improvement at 40 weeks of adalimumab therapy. D) Histopathological features of cutaneous Crohn's disease: Low power magnification showing a diffuse non-caseating granulomatous inflammatory infiltrate with pseudoepitheliomatous hyperplasia (hematoxylineosin stain; original magnification $\times 40$ ). Close-up view showing a granulomatous infiltrate with multinucleated giant cells (hematoxylin-eosin stain; original Magnification $\times 200$ ). 
We report a case of cutaneous perianal CD with a pseudocondylomatous appearance mimicking genital warts, highlighting the importance of early diagnosis and appropriate treatment of atypical perianal CD. To our knowledge, this case represents the third report of granulomatous cutaneous $C D$ treated with adalimumab, supporting its efficacy in therapy-resistant patients.

Acknowlegements. Conflict of interest: none. Funding sources: none

${ }^{1}$ Dermatology Department, Hospital
Santo António dos Capuchos,
Centro Hospitalar de Lisboa-
Central, 1169-050 Lisbon, Portugal
${ }^{2}$ Gastroenterology Department,
Hospital Santo António dos
Capuchos, Centro Hospitalar de
Lisboa-Central, Lisbon, Portugal
<saralestre@gmail.com>

Sara LESTRE ${ }^{1}$ Jaime RAMOS ${ }^{2}$ Alexandre JOÃO ${ }^{1}$ Vasco SERRÃO ${ }^{1}$

1. Eames T, Landthaler M, Karrer S. Crohn's disease: an important differential diagnosis of granulomatous skin diseases. Eur J Dermatol 2009; 19: 360-4.

2. Ingle SB, Loftus Jr EV. The natural history of perianal Crohn's disease. Dig Liver Dis 2007; 39: 963-9.

3. Palamaras I, El-Jabbour J, Pietropaolo N, et al. Metastatic Crohn's disease: a review. J Eur Acad Dermatol Venereol 2008; 22: 1033-43.

4. Asma SD, Soumaya Y, Kahena J, Raouf DM. Nejib D. Perianal Crohn disease. Dermatol Online J 2006; 12: 18.

5. Garg M, Kawsar M, Forster GE, Medows NJ. Perianal Crohn's disease masquerading as perianal warts. Sex Transm Infect 2002; 78: 302-3.

6. Miller FA, Jones CR, Clarke LE, Lin Z, Adams DR, Koltun WA. Successful use of adalimumab in treating cutaneous metastatic Crohn's disease: Report of a case. Inflamm Bowel Dis 2009; 15: 1611 -2. doi: $10.1684 /$ ejd.2010.0975

\section{Sarcoidosis expressing as four distinct cutaneous lesions}

We herein report a patient with sarcoidosis who exhibited four different cutaneous forms and livedo. A 62-year-old Japanese woman had noticed reddish papules on her nose eight years previously (figure 1A). She also had a two year history of uveitis. Asymptomatic nodules appeared in her knee (figure $1 B$ ). She noticed several diffuse and indurated erythemas on both hands (figure $1 C$ ) and plaque lesions with livedo reticularis on both upper thighs (figure 1B). A skin biopsy specimen from her nose demonstrated dense naked granulomatous infiltrates (figure 1D). The histopathological findings of livedo showed thin-walled, narrowly dilated small arteries and epithelioid granulomas in the lower dermis and subcutaneous tissues (figure 1E). A microscopic examination using polarized light did not reveal the presence of silicon or a foreign body in the granulomas. PAS and Ziehl-Neelsen staining did not demonstrate the presence of bacilli.

Laboratory studies revealed an increased serum lactose dehydrogenase level $(276 \mathrm{U} / \mathrm{mL})$, angiotensin I converting enzyme value $(34.7 \mathrm{U} / \mathrm{mL}$, normal range within $21.4 \mathrm{U} / \mathrm{mL}$ ), and sialylated carbohydrate antigen KL-6 level $(628 \mathrm{U} / \mathrm{mL})$; other values, including the peripheral blood counts and liver and renal function parameters, were normal. A tuberculin test was negative. Chest radiography showed multiple miliary and infusion shadows (figure $1 F$ ). A gallium-67 scan revealed hepatosplenomegaly. The echocardiography results were normal. We diagnosed her as having polymorphous cutaneous sarcoidosis including nodular and lupus pernio, infiltrating
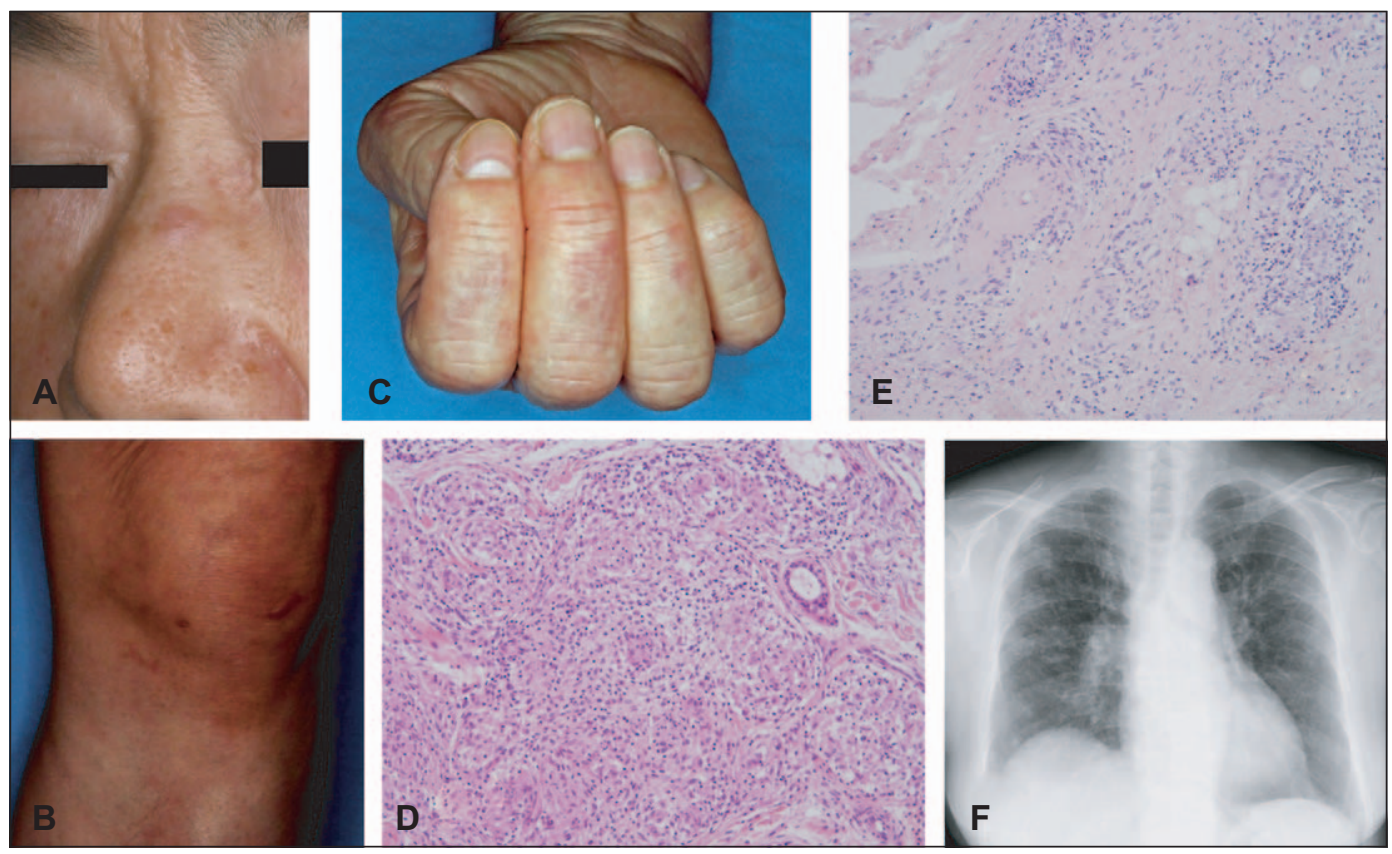

Figure 1. A) Nodule on the nose. B) Scar sarcoidosis and livedo reticularis on the left knee. C) Diffuse and infiltrative erythema (lupus pernio) on the right hand. D) Histopathological findings showing naked granulomatous infiltrates in the dermis (hematoxylin and eosin staining; original magnification, $\times 40$ ). E) Thin-walled, small arteries in fat tissues. The skin biopsy specimens were taken from the livedo lesions on the knee (original magnification, $\times 100$ ). F) Chest X-ray showing multiple miliary shadows. 